\title{
Falsches Risikomanagement
}

\author{
Hans-Gert Heuft Rainer Blasczyk \\ Institut für Transfusionsmedizin, Medizinische Hochschule Hannover, Deutschland
}

Die möglichst geringe Spenderexposition ist ein weltweiter transfusionsmedizinischer Standard und einer der wesentlichen Voraussetzungen für die Nachhaltigkeit des medizinischen und technischen Fortschritts in der Transfusionsmedizin. So ist die reduzierte Spenderexposition ein wichtiges Argument für die Gewinnung von bis zu 3 Einheiten gefrorenen Frischplasmas (GFP) im Rahmen einer präparativen Plasmapherese, für die Bereitstellung von bis zu 4 Teilmengen aus einem Erythrozytenkonzentrat (EK), GFP oder Thrombozytenkonzentrat (TK) für Frühgeborene, Säuglinge und Kleinkinder oder für die Gewinnung mehrerer verschiedener Blutkomponenten wie EK, GFP und TK im Rahmen einer Einzelspender-Multikomponentenspende. Die Bereitstellung von Gerinnungsfaktoren als Kryopräzipitate aus Mini-Pools von 12 Spendern hat in Großbritannien in den 1980er Jahren vielen Hämophilen das Leben gerettet, während die hochangereicherten Faktor-VIII-Konzentrate aus großen Plasma-Pools von 20000 Spenden auf dem europäischen Kontinent und in Nordamerika eine große Hepatitis-C- und HIV-Epidemie unter den Betroffenen ausgelöst hat [1].

In ihren aktuellen Arbeiten vollziehen Andreas Greinacher und Kollegen einen gefährlichen Paradigmenwechsel. Sie stufen in zwei an unterschiedliche medizinische Fachgebiete gerichteten Publikationen in Transfusion Medicine and HeMOTHERAPY (Langfassung, 15 Seiten) [2] bzw. in der Deutsche Medizinische Wochenschrift (Kurzfassung, 5 Seiten) [3] TK von 4-6 Vollblutspenden als gleichwertig gegenüber Einzelspender-Apherese-TK ein. Als einziges Entscheidungskriterium für Pool-TK oder für Apherese-TK wird in beiden Publikationen die «lokale Verfügbarkeit» genannt. Unkommentiert wird in der Langfassung noch erwähnt, dass die Verabreichung von Pool-TK mit einer höheren Refraktärität verbunden ist als die von Apherese-TK [2]. Es ist bemerkenswert, dass dieser für Therapieentscheidungen bedeutsame Unterschied in der Kurzfassung [3] fehlt. Sollte dem breiten ärzt- lichen Anwenderkreis verschleiert werden, dass Pool-TK zumindest für Thrombozyten-Langzeitempfänger Produkte zweiter Wahl sind?

Aus guten Gründen fehlen in den britischen «Guidelines for the Use of Platelet Transfusions» Entscheidungskriterien für eines der beiden Thrombozytenpräparate [4, p. 11]. Diese Frage ist keineswegs so gut untersucht, dass sie in einem Konsensuspapier mehrerer Fachgesellschaften auf die «lokale Verfügbarkeit» reduziert werden kann. In den britischen Guidelines findet sich aber der mehr als nahe liegende Hinweis, dass Pool-TK die Spenderexposition deutlich erhöhen. Welches Ausmaß unterschiedlicher Spenderexpositionen in Kauf genommen werden würde, wenn Pool-TK anstelle von Apherese-TK eingesetzt werden würden, zeigen beispielhaft aktuelle Zahlen aus der Medizinischen Hochschule Hannover. Im Jahre 2006 erhielten 221 Patienten während Ihres stationären Aufenthalts 1-3 Apherese-TK, 3 EK und im Median auch nur 3 (0-14) GFP; sie hatten also Kontakt mit Blut einer überschaubaren Gruppe von 9 Spendern. Bei Einsatz von Pool-TK mit der Mindestspenderzahl von 4 Spenden hätte sich die Spenderexposition bei diesem Patientenkollektiv auf 18 Spender erhöht. Auf der anderen Seite fanden sich in der Medizinischen Hochschule Hannover im Jahre 2006131 polytransfundierte Patienten, die mindestens 20, im Median 34 (20-178), Apherese-TK erhalten hatten. Diese Gruppe erhielt zusätzlich im Median 34 (3-247) EK und 32 (0-233) GFP. Auch in dieser polytransfundierten Gruppe würde sich die mediane Spenderexposition bei 4 Spenden pro Pool-TK von 100 auf 202 Spender verdoppeln. Insgesamt wurden im Jahre 2006 an der Medizinischen Hochschule Hannover 11476 AphereseTK von 1342 Spendern bei 1821 Patienten angewendet $(0,74$ Spender pro TK-Empfänger). Bei ausschließlicher Verwendung von Pool-TK aus 4 Einzelspenden wären zur Versorgung der Patienten der Medizinischen Hochschule Hannover 45904 Einzelspenden erforderlich gewesen. Dies würde bei

\begin{tabular}{ll}
\hline KARGER & @ 2007 S. Karger GmbH, Freiburg \\
Fax +49 7614520714 & Accessible online at: \\
$\begin{array}{l}\text { E-mail Information@Karger.de } \\
\text { www.karger.com }\end{array}$ & www.karger.com/tmh
\end{tabular}


durchschnittlich 1,8 Spenden pro Vollblutspender und Jahr 25502 individuellen Spendern entsprechen (14 Spender pro TK-Empfänger). Die Spenderexposition wäre daher für TK-Empfänger der Medizinischen Hochschule Hannover auf das 19 fache angestiegen.

Das Einzelspender-Blutprodukt ist ein einfacher und effektiver Mechanismus zur Risikominimierung für Transfusionsempfänger. Dieser Gold-Standard wird jetzt unüberlegt und ohne Not infrage gestellt. Die Transfusionsmedizin hat in den vergangenen Jahrzehnten viel unternommen, um Risiken zu minimieren. Das reicht vom Ausschluss bestimmter Spendergruppen (z.B. homosexuelle oder bisexuelle Männer, isoliert anti-HBc-positive Spender) über verschärfte Spender-Reiseanamnesen (West-Nil-Virus in den USA) bis hin zur drastischen Verschärfung der Screening-Anforderungen auf transfusionsrelevante Viren (NAT-Untersuchungen). Die Reduktion des Infektionsrisikos für die klassischen transfusionsrelevanten Viren HBV, HCV und HIV, sollte nicht dazu führen, das Risikomanagement zu vernachlässigen. Die aktuelle Anordnung des Paul-Ehrlich-Instituts zur Verschärfung der Spender-Reiseanamnese für Teile Afrikas, Südostasiens sowie Inseln im Indischen Ozean wegen der Chickungunya-Fieber-
Epidemie passt genauso in dieses Bild wie die schon lange bestehende Vorschrift des Paul-Ehrlich-Instituts, in allen Gebrauchs- und Fachinformationen zu Blutprodukten den Warnhinweis auf noch unbekannte Erreger aufzunehmen. In dieser Situation ist das Poolen von Blutprodukten mit der Folge, dass sich die Spenderbelastung von gering transfundierten und polytransfundierten TK-Empfängern vervielfacht, ein dramatischer Hinweis für ein verfehltes Risikomanagement. Wir fragen uns, welches Thrombozytenprodukt die Autoren dieses Leitlinienentwurfs auswählen würden, wenn sie selbst oder ihre Angehörigen betroffen wären.

\section{Literatur}

Levine PH: HIV infection in hemophilia. J Clin Apher 1993;8:120-125.

2 Greinacher A, Kiefel V, Klüter C, Kroll H, Pötzsch B, Riess H: Empfehlungen zur Thrombozytentransfusion der Thrombozytenarbeitsgruppe der DGTI, GTH und DGHO. Transfus Med Hemother 2006;33:528-543.

3 Greinacher A, Kiefel V, Klüter C, Kroll H, Pötzsch B, Riess H: Empfehlungen zur Thrombozytentransfusion der Thrombozytenarbeitsgruppe der DGTI, GTH und DGHO. Dtsch Med Wochenschr 2006;131:2675-2679.

4 British Committee for Standards in Haematology, Blood Transfusion Task Force: Guidelines for the use of platelet transfusions. Br J Haematol 2003;122:10-23.

\section{Erratum}

In the article

\section{«A Prospective Crossover Trial Comparing Performance and in vitro Platelet Quality of Three New Aphesesis Devices"}

(Transfus Med Hemother 2006;33:520-527) one unit in table 5 and in the footnote of table 5 accidentally was given wrong. The correct unit for glucose consumption is $10^{-10} \mathrm{mg} / \mathrm{PLT}$. We apologize for this mistake. 